\title{
Archipel
}

ARCHIPEL Études interdisciplinaires sur le monde insulindien

$95 \mid 2018$

Varia

\section{Utuy Tatang Sontani et l'homme aux yeux de braise}

Utuy Tatang Sontani and the man with smouldering eyes

\section{Henri Chambert-Loir}

\section{(2) OpenEdition}

Journals

Édition électronique

URL : https://journals.openedition.org/archipel/680

DOI : 10.4000/archipel.680

ISSN : 2104-3655

Éditeur

Association Archipel

Édition imprimée

Date de publication : 29 juin 2018

Pagination : 111-132

ISBN : 978-2-910513-79-5

ISSN : 0044-8613

Référence électronique

Henri Chambert-Loir, "utuy Tatang Sontani et l'homme aux yeux de braise », Archipel [En ligne], 95 | 2018, mis en ligne le 01 juillet 2018, consulté le 27 août 2021. URL : http://journals.openedition.org/ archipel/680 ; DOI : https://doi.org/10.4000/archipel.680 


\title{
VARIA
}

\author{
HENRI CHAMBERT-LOIR ${ }^{1}$
}

\section{Utuy Tatang Sontani et l'homme aux yeux de braise}

En septembre 1965, le 27 précisément, Utuy Tatang Sontani partit en voyage. Il avait 45 ans. Sa carrière d'écrivain l'avait comblé jusque là : son roman, ses nouvelles et ses pièces de théâtre le classaient parmi les écrivains les plus éminents de sa génération, il siégeait à la direction de la plus grande organisation culturelle du pays, il était respecté, connu, admiré ; l'avenir était plein de promesses. Et pourtant, lorsqu'il monta dans l'avion de Pékin, ce 27 septembre, sa carrière prit fin subitement.

Utuy est né en 1920, à Cianjur, une bourgade située à mi-chemin entre Bogor et Bandung, réputée pour ses traditions artistiques. Il commença à écrire très tôt dans sa langue maternelle, le soundanais. Lorsque les Japonais envahirent l'Indonésie sans rencontrer grande résistance, en 1942, Utuy avait déjà publié, en feuilleton, en soundanais, un gros roman historique, Tambera, ainsi qu'une longue nouvelle «Mahala Bapa » (Tuer le père). Il avait fait une très courte scolarité dans sa ville natale : trois ans d'école primaire et quelques années de plus au Taman Siswa, l'école fondée par Ki Hadjar Dewantara, qui proposait un enseignement d'esprit et de culture indigènes face à l'enseignement occidental des écoles fondées par le gouvernement colonial néerlandais. Par la suite, il ne passa que quatre mois au Taman Dewasa de Bandung. Grâce au Taman Siswa, c'est sans difficulté, comme il le raconte ci-dessous, qu'il se mit à écrire en indonésien, lorsque les Japonais interdirent l'usage du néerlandais et des langues régionales. Il rédigea une nouvelle version, indonésienne cette

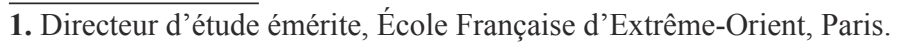


fois, du roman Tambera, puis écrivit des pièces de théâtre qui, mises en scène et publiées au long des années 1950, lui valurent le succès.

Utuy a peu écrit : un roman, moins de vingt nouvelles (cerpen) et sept pièces de théâtre ${ }^{2}$. Il a néanmoins gagné une place de premier plan sur la scène littéraire indonésienne, et ses pièces ont été jouées avec un grand succès. Il les a aussi publiées sous forme narrative, de sorte à en faire des nouvelles (cerpen). Les quelques analyses de son oeuvre (Rosidi, 1959 ; Santa Maria, 1965 ; Jassin, 1967 ; Teeuw, 1979 ; Aveling, 1979) soulignent son individualisme, sa sympathie pour les déshérités et sa tendance à décrire des personnages masculins faibles confrontés à des personnages féminins forts. À l'étranger également, tout au moins en Chine et en URSS, il était tenu en haute estime. Lorsqu'il arriva à Pékin, fin septembre 1965, il fut reçu comme un écrivain célèbre et traité comme un hôte de marque.

Il faisait partie de l'énorme délégation indonésienne, environ 500 membres, venue assister au seizième anniversaire de la proclamation de la République Populaire, mais il appartenait en fait à la « délégation malade» (delegasi sakit) de ceux venus en Chine pour se soigner. Après la célébration du $1^{\text {er }}$ octobre, il fut envoyé à l'hôpital de Canton pour plusieurs mois. La masse des réfugiés indonésiens, tous ceux qui n'avaient pas pu rentrer en Indonésie après les événements du 30 septembre, se trouva sous l'autorité d'une Délégation autoproclamée du comité central du parti communiste indonésien (Delegasi CC PKI) et fut traitée en bloc par le gouvernement chinois. La révolution culturelle, qui commença justement en 1966, aggrava la situation. Elle imposait, entre autres conséquences, que les écrivains autrefois admirés, comme Utuy, fussent désormais condamnés comme petits bourgeois décadents et interdits. Les Indonésiens furent logés dans un camp, à Nancang, étroitement gardé et dont ils n'avaient pas le droit de sortir. Utuy évoque, dans la très longue nouvelle qui clôt le volume Di Bawah Langit Tak Berbintang (Sous un ciel sans étoiles, 2001), une partie de ses tribulations, d'un hôpital et d'un camp à un autre. Il se brouilla très tôt avec la Délégation et se retrouva ostracisé par la communauté indonésienne en Chine. Il éprouvait du mépris pour ses compagnons d'infortune, qu'il traite dans ses écrits d' « indigènes » (inlander).

Pour la plupart des réfugiés indonésiens, cet épisode chinois dura entre dix et quinze ans, et il fut pour beaucoup d'entre eux une expérience difficile. Cette période est très peu documentée, du fait notamment que les intéressés eux-mêmes sont restés extrêmement avares d'informations dans les ouvrages autobiographiques, pourtant nombreux, qu'ils ont rédigés par la suite.

En 1973, Utuy obtint la permission d'aller se soigner aux Pays-Bas. Mais, en route, après une semaine de train, il fit halte à Moscou, où il fut

2. Aveling (1979) donne un résumé et une analyse de toutes les oeuvres d'Utuy publiées avant 1965. On trouvera la traduction de deux nouvelles du recueil Orang-Orang Sial dans l'anthologie de Denys Lombard (1968) et dans le recueil Vigile (2009). 
si chaleureusement accueilli qu'il décida d'y rester. La communauté des réfugiés indonésiens, beaucoup plus réduite qu'en Chine, était administrée par un Comité extérieur du parti communiste indonésien (Comite Luarnegeri $\mathrm{PKI}),{ }^{3}$ mais Utuy était une figure exceptionnelle et reçut un traitement de faveur du régime soviétique : fêté comme un écrivain éminent, il fut logé dans un grand appartement, assisté par un fonctionnaire et bénéficia des services d'un traducteur (Vilen Sikorsky, qui devint un ami proche). Il fut aussi chargé de cours d'indonésien à l'Université d'État de Moscou, en tant qu'assistant de la linguiste Lyudmila Demidyuk.

Son cinquante-cinquième anniversaire, en 1975, fut fêté avec faste à la Bibliothèque des langues étrangères, et une bibliographie de ses oeuvres parut en 1977 (Supartono, 2001 : 151). Mais ceci marquait la fin de sa gloire. Comme il l'avait fait en Chine avec la Délégation, Utuy refusa de coopérer avec le Comité de Moscou, avec la même conséquence : il se retrouva peu à peu isolé et déconsidéré. Il déménagea pour un appartement plus petit, les invitations se firent plus rares, l'assistant disparut. C'est à cette époque qu'il entra en contact avec Denys Lombard, auquel il envoya une partie des textes qu'il avait écrits en URSS, dont l'un, la nouvelle " Pourquoi j'écris », fut publié en traduction française dans la revue Archipel (volume 15), en 1978.

Déjà, en 1975, sa situation n'était pas brillante. Ajip Rosidi relate dans ses mémoires (2008 : 602-604, avec une photo p. 633) la visite qu'il lui rendit cette année-là, dans un hopital de Moscou, conduit par Sikorsky. Utuy, malade et déprimé, lui confia qu'il désirait que sa femme le rejoigne, mais celle-ci, contactée par Ajip à son retour à Jakarta et qui était sans nouvelles depuis des années, n'avait aucun désir d'aller en URSS ${ }^{4}$. Waloejo Sedjati, un autre réfugié politique indonésien, qui devait finalement émigrer en France, passa les années 1975-1982 à Moscou, où il rencontra Utuy à plusieurs reprises. Il lui tint même compagnie durant deux semaines, dans une maison de repos à l'écart de Moscou. Il lui consacre un court chapitre de ses mémoires (2013 : 257-264). Le portrait qu'il en donne est amical, mais n'est guère flatteur. Utuy est aigri, susceptible et colérique.

Pour finir, il meurt dans l'isolement, à Moscou, en septembre 1979. Au peintre Kuslan Budiman, qui s'était beaucoup occupé de lui sur la fin de sa vie, Utuy confia son désir d'être enterré en musulman. Ajip Rosidi (2001 : 20) rapporte un propos d'Utuy que lui a communiqué Kuslan Budiman : " Quoi qu'il en soit, je suis musulman et mon grand-père était haji. » Il fut enterré dans le quartier Tatar d'un cimetière situé à quelque distance de Moscou (Sedjati, 2013 : 263).

3. La situation des réfugiés indonésiens en URSS est mieux documentée que celle de Chine ; voir l'article de David Hill (2014).

4. Ajip avait raconté cette rencontre plus succinctement dans sa préface de $2001:$ 13-14. 
Utuy a écrit deux romans durant les sept années qu'il a passées en Chine (Benih et Tumbuh, Graine et Croissance ; cf. Supartono, 2001 : 153). Il a été plus productif en URSS, où il a écrit plusieurs romans et récits, durant les trois dernières années de sa vie. Alex Supartono, qui est l'auteur d'un excellent article sur ces oeuvres et le contexte de leur rédaction (2001), donne un aperçu de deux romans (Kolot Kolotok et Pemuda Telanjang Bulat, Immaturité et Le Jeune homme nu, demeurés inédits ${ }^{5}$; plus un roman inachevé, Melodi yang Belum Selesai, Mélodie inachevée) et de quatre récits autobiographiques. Ceux-ci ont été publiés par Ajip Rosidi, réunis sous le titre du plus long d'entre eux : Di Bawah Langit Tak Berbintang (2001). Ajip Rosidi mentionne également une pièce de théâtre : Bukan Orang Besar (Un Homme ordinaire), qu'il qualifie de « propagande bon marché » et une nouvelle : «Anjing » (Un Chien), qui fut publiée dans un autre recueil posthume.

La nouvelle Di Bawah Langit Tak Berbintang est un récit ambigu et intrigant sur les années chinoises. Tous les autres textes se rapportent à l'Indonésie antérieure à 1965. Comme tous les autres exilés politiques indonésiens, Utuy ne s'intéresse pas à son nouveau cadre de vie : il écrit en indonésien sur la situation indonésienne, à l'intention du public indonésien. L'un des textes autobiographiques, traduit ci-dessous, dresse un portrait, surprenant à plus d'un titre, d'un personnage connu. Afin de conserver l'effet de surprise voulu par l'auteur, l'analyse de la nouvelle suivra sa traduction. Signalons seulement que plusieurs éléments du récit sont des allusions à des personnages féminins des deux textes précédents : Onih, qui apparaît dès la première phrase et est citée une fois encore plus loin, est la protagoniste du second récit, auquel réfèrent également le refrain "I belong to you, you belong to me ", de même que les " émotions par le biais des femmes", tandis que les jeunes filles « jacassant en néerlandais », ainsi que « ma voisine de Cianjur », sont des allusions au premier récit. Voici donc la nouvelle en question, traduite de l'indonésien.

\section{UTUY TATANG SONTANI WHAT'S IN A NAME ?}

Si l'occupation japonaise avait fait disparaître Onih je ne sais où, c'est alors, au contraire, que mon nom commença à briller au firmament des écrivains. Durant cette période, l'enseignement du néerlandais fut supprimé dans les écoles et fit place à l'obligation d'apprendre l'indonésien ; les journaux en langues régionales durent mettre la clef sous la porte, tandis que les écrivains et les journalistes, les vieux comme les jeunes, durent se résoudre à écrire

5. Kolot Kolotok a cependant été traduit en russe par Vilen Sikorsky et publié, en 1988, dans une grosse anthologie de littérature indonésienne des années 70 . 
en indonésien s'ils voulaient continuer à travailler. Pour ma part, passer du soundanais à l'indonésien n'était pas un problème : l'indonésien, il y a longtemps que je l'avais appris au Taman Siswa. C'est donc tout naturellement que j'envoyai des poèmes dans cette langue à la revue Panca Raya, l'unique revue culturelle du pays à ce moment-là ${ }^{6}$.

Ce que je n'avais pas prévu, c'est que, du fait qu'un de mes poèmes avait eu la chance de paraître dans cette revue, je reçus un jour une invitation du Gunseikanbu ${ }^{7}$ pour assister à un congrès culturel, à Jakarta. L'invitation s'accompagnait d'un billet de train de seconde classe.

Mais voyez un peu comment ça s'est passé ! Pour commencer, je me suis trompé de train : je devais prendre l'express, mais je suis monté dans un omnibus. Une fois arrivé, je n'ai pas cherché comme les autres un hôtel de luxe, mais j'ai dû me contenter d'un petit hôtel dans une ruelle. Et pendant le congrès, je n'ai évidemment pas prononcé un discours, un rapport ou une communication, non, tout mon être était uniquement rempli d'une admiration sans borne, parce que, là, pour la première fois, je voyais de mes propres yeux les hommes nommés Sanusi Pane, et Armijn Pane, et Sutan Takdir Alisjahbana, les illustres écrivains du groupe Pujangga Baru. Et là aussi, je fus présenté à des peintres fameux, des compositeurs renommés et des acteurs de cinéma célèbres.

Il se produisit un jour un incident que je ne peux oublier. Je lus sur une porte la pancarte " Section littéraire » et je m'enhardis à entrer. Une réunion se tenait, sous la présidence d'Armijn Pane. Voyant une chaise libre dans le fond de la pièce, je m'y installai. Armijn Pane, qui était en train de parler, s'interrompit brusquement, me lança un regard et prononça sur un ton sans réplique : «Votre place est dehors!»

Il ne me restait qu'à sortir - avec une question en tête, une question qui demeure irrésolue jusqu'à aujourd'hui : n'avais-je pas été autorisé à écouter leur délibération parce que j'étais jeune et ignorant ? Mais dans ce cas, pourquoi avais-je été invité ?

Tout de même, un résultat de ma présence à ce congrès qui dépassait les limites de mon entendement, fut ma rencontre avec un de mes anciens professeurs du Taman Dewasa. Dès qu'il m'aperçut, il me fit des compliments et me déclara : " On va ouvrir une agence du Putera pour Priangan à Bandung. Je serai directeur de la section culturelle, et je t'embaucherai. »

Il tint sa promesse. Aussitôt l'agence ouverte, on me fit quitter le bureau de la Préfecture, où j'avais travaillé jusque là pour le maigre salaire de 17,50 florins par mois, pour m'employer au Putera pour 25 florins $^{8}$. Mais ce n'était

6. Alex Supartono (2001 : 137) note qu'Utuy fait erreur avec Panji Pustaka, la revue Panca Raya n'ayant commencé à paraître qu'en 1945.

7. Gouvernement militaire d'occupation japonaise.

8. Pendant les deux premières années de l'occupation japonaise, la monnaie en vigueur consistait 


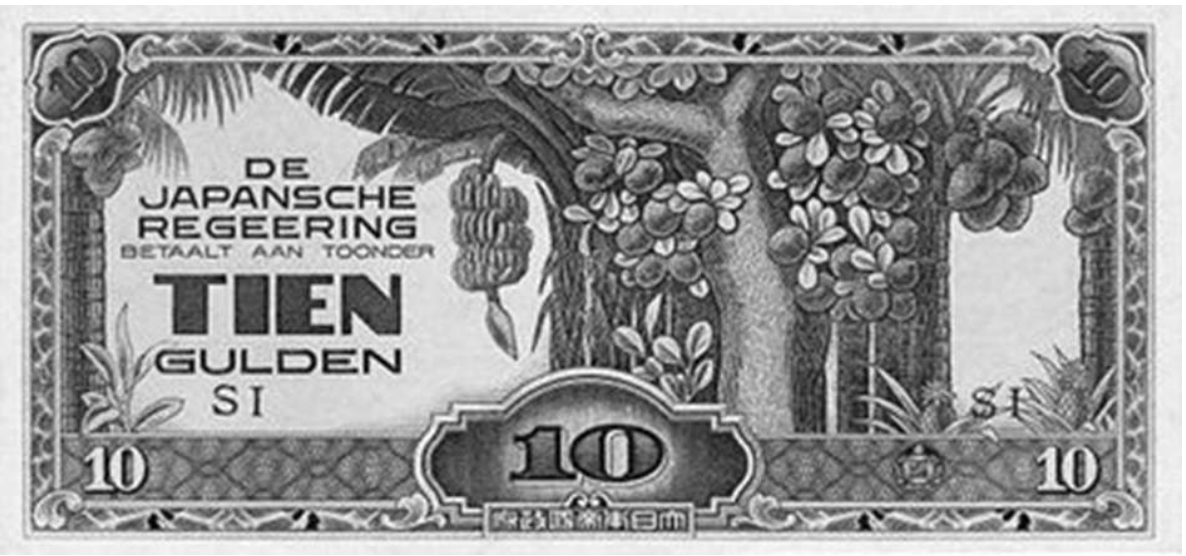

Fig. 1 - Billet de 10 florins émis par le gouvernement japonais d'occupation, à Java, en 1942. Cette monnaie était appelée par les Indonésiens oeang Djepang, ou oeang pisang à cause du dessin d'un bananier sur ce billet. (National Museum of American History, cliché de Godot13.)

pas une question de salaire, vous devez le comprendre. Il vous faut savoir que Putera n'était pas un nom comme les autres : c'était l'abréviation de Pusat Tenaga Rakyat (Centrale des forces populaires), un mouvement qui réunissait les forces populaires des milieux les plus divers, depuis le sport jusqu'à la culture et même la politique, un mouvement dirigé par Bung Karno, Bung Hatta, Ki Hajar Dewantara et Kiai Haji Mas Mansur, ceux-là mêmes qui étaient alors connus comme « les quatre inséparables ». Aussi, vous vous en doutez, les gens employés par le Putera n'étaient pas n'importe qui ; la société, en tout cas, les regardait comme des personnages importants.

Moi-même, comment ne me serais-je pas senti gonflé d'importance, même au petit pied ? Pratiquement chaque semaine, on pouvait lire mes écrits poèmes ou articles - dans le journal Cahaya (Lumière), l'unique journal paraissant à Bandung. En plus, je fus nommé président de la branche de Priangan des Écrivains de la Nouvelle Génération (Sasterawan Angkatan Baru), assisté par un secrétaire, un jeune poète du nom de Kelana Asmara. Mon travail ne consistait pas à rester simplement assis devant un bureau : je voyageais de temps à autre à travers la province, pour diriger une représentation théâtrale ou pour accompagner une troupe de wayang golek ou de reog ${ }^{9}$. Et

en florins (gulden) émis par le gouvernement militaire japonais, dont les billets étaient inscrits en néerlandais (voir la fig. 1). La mention du Putera date précisément la situation, car cette institution fut créée par les Japonais en avril 1943 et dissoute au bout d'un an.

9. Le wayang golek, populaire surtout en pays soundanais, est un théâtre de marionnettes comparable au théâtre d'ombres (wayang kulit) du pays javanais, mais dont les figurines sont en ronde bosse et sont donc vues du public. Le reog soundanais, très différent du reog de Java Est, est un art populaire mêlant danse, musique, chanson et narration ; joué traditionnellement 
par-dessus tout, on me donnait le temps d'écrire. Je tirai le meilleur parti de cette occasion pour adapter mon Tambera en indonésien.

Vous vous demandez, bien sûr, ce que devenait ma quête d'émotions par le biais des femmes?

Avant que je réponde à cette question, vous devez savoir que le regard sur les femmes change inévitablement d'une époque à l'autre. Au temps de l'occupation japonaise, la jeune fille prisée comme une fleur assaillie par les bourdons n'était plus celle capable de jacasser en néerlandais. Non, à ce momentlà, suite à l'enseignement du dynamisme japonais qui avait été dispensé à la nation indonésienne, la jeune fille brillante aux yeux des jeunes gens était celle dont on disait qu'elle avait « un visage ardent », celle qui osait débattre avec les hommes, qui avait le courage de prendre la parole dans les réunions, et plus admirable encore, celle qui savait faire un discours devant une foule.

Vous comprenez bien que mon idéal du moment n'était pas de savoir comment trouver une femme qui puisse me tenir compagnie en chantant « $I$ belong to you, you belong to me », mais comment affronter avec elle tempêtes et ouragans comme les deux ailes d'un aigle. Cet idéal, Kelana Asmara, mon plus proche compagnon de lutte, l'avait formulé lui aussi.

Pour moi, heureusement, trouver la jeune fille de mes rêves ne revenait pas à chercher une aiguille dans la nuit noire, car une telle créature se trouvait justement dans le bureau du Putera. Elle avait été recrutée du fait de son ascendance, car elle était fille de combattant : son père faisait partie des nationalistes qui avaient mené une lutte acharnée contre les Hollandais. Du point de vue physique, elle n'était pas aussi jolie que ma voisine de Cianjur ; elle était même bien inférieure à Onih. Mais son ardeur, son audace dans les débats avec les hommes et son courage à parler en public, c'est cela qui lui avait valu le sobriquet de « la fleur du Putera ». Non seulement les jeunes gens, mais les hommes mariés aussi la regardaient avec attention.

Mais pourquoi aurais-je dû m'inquiéter ? Ma mère et la sienne s'étaient secrètement promis que nous serions leur bru et leur gendre. Elle-même, en dépit de son goût pour la discussion, s'appliquait toujours à n'employer avec moi que des mots modérés. Notre chef de service, qui était au courant de tout, avait l'habitude de nous taquiner, nous demandant quand nous formerions les deux ailes d'un aigle.

Ce que je ne pouvais pas prévoir, c'est que le chef de service annonça un beau jour qu'un nouvel employé, venu de Jakarta, allait rejoindre le bureau. Le plus étrange est que ce personnage était, disait-on, un favori de Bung Hatta. Rendez-vous compte ! Un jeune qualifié à cette époque de favori de Bung Hatta devait pour le moins avoir hérité de son âme de combattant et il avait sans aucun doute droit au qualificatif de jeune homme « au visage ardent ».

par quatre hommes, le reog mêle comique et critique sociale. 
Lorsque le nouvel employé se présenta, le lendemain, les prévisions se trouvèrent confirmées : qu'il fût un « jeune au visage ardent» se voyait clairement dans ses yeux de braise, ses mouvements brusques et sa voix monocorde, sans intonation. Toute sa posture devant moi me fit penser au personnage de Kawista dans le roman Tambera que j'étais en train d'écrire, ce protagoniste à l'opposé de Tambera, si noble et raffiné, ce Kawista grossier et intransigeant qui, du fait de son tempérament, choisit de s'opposer aux Hollandais de façon radicale et aboutit à une défaite qui provoqua l'anéantissement de la population de Banda et son propre exil.

«Si je m'identifie à Tambera, le noble, comment pourrai-je me lier d'amitié avec lui, qui est aux antipodes de ce personnage ?»-telle est la réflexion que je me fis en lui serrant la main.

Un sentiment étrange s'imposa à moi, cependant. C'est lorsqu'il prononça son nom. Qu'y avait-il de bizarre ? C'est que j'étais certain que le nom qu'il annonçait ne lui avait pas été donné par ses parents ; c'est un nom qu'il avait créé lui-même, de même que mon propre nom ou celui de Kelana Asmara. Seulement, si mon nom peut assurément être qualifié de mélodieux et celui de Kelana Asmara de romantique, celui qu'annonçait le jeune homme aux yeux de braise clamait avec force un esprit d'héroïsme.

« Bah, l'esprit d'héroïsme qui émane de lui ne sera pas supérieur à celui de Kawista », conclus-je. Et à partir de là, bien que lui et moi parlions rarement - à l'image des deux héros de mon roman -, je finis peu à peu par observer de loin ses mouvements et à pénétrer secrètement son esprit à travers ses gestes et ses paroles.

Comment aurions-nous pu parler fréquemment, de toutes façons? Il venait rarement au bureau, et toujours en retard; il s'asseyait un instant au bureau du gardien et l'aidait à faire des enveloppes, puis il repartait on ne sait où. Le chef de service le laissait peut-être en faire à sa guise parce qu'il était réputé être un favori de Bung Hatta, je ne sais pas ; ce qui est sûr, c'est que son comportement désinvolte me renforçait dans la conviction qu'il était exactement l'homme qu'il me fallait pour dépeindre le personnage sans manières de Kawista.

Je ne me souviens plus depuis combien de temps il était parmi nous, lui et son arrogance, lorsqu'un jour se produisit un événement stupéfiant. Chaque matin, avant de se mettre au travail, les employés du Putera avaient l'habitude de faire une séance de gymnastique taiso avec les Japonais du bureau Sendenbu ${ }^{10}$, qui se trouvait à proximité. Mais ce matin-là, seuls quelques employés du Putera, dont moi-même, étaient présents pour le taiso. Les autres ne participaient pas parce qu'ils n'étaient pas encore arrivés. Voyant cela, le chef du Sendenbu se mit en colère. Il fit venir tous les employés, depuis le chef de service jusqu'au gardien, et les fit mettre en rangs. Je me souviens de la

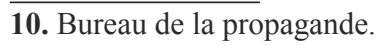


pâleur de tous, bien compréhensible, car le chef du Sendenbu qui leur donnait des ordres était un officier japonais en grand uniforme et portant un sabre de samouraï.

« Pourquoi ne voulez-vous pas faire le taiso ce matin? », aboya-t-il. « D'où vient cette indiscipline?»

Tout le monde resta silencieux; pas un seul ne répondit.

« De qui vient cette idée de rébellion? », insista-t-il avec colère.

À la surprise de tous, au milieu de cette atmosphère insoutenable, le jeune homme aux yeux de braise s'avança soudain et déclara en se frappant la poitrine : "Moi ; c'est moi qui ai incité nos camarades à ne pas faire le taiso. »

«Et pourquoi ? », demanda le Japonais, les yeux exorbités.

«Parce que nous ne sommes pas des employés ordinaires. Nous sommes des dirigeants. Notre travail n'est pas dicté par des horaires, il n'est pas soumis au jour et à la nuit. Et donc, si nous arrivons en retard au bureau et ne pouvons participer au taiso, ce n'est pas par paresse, mais parce que nous travaillons la nuit, et il arrive que nous devions aussi travailler au réveil. Nous ne refusons pas le taiso, nous le faisons à la maison, avant de venir au bureau. »

Entendant cette réponse, l'officier japonais, je ne sais pas pourquoi, ne prononça plus un mot. Et depuis lors, la pratique du taiso du matin fut laissée à notre bon vouloir.

À la suite de cette histoire, vous vous en doutez, je découvris dans le jeune homme aux yeux de braise des signes de supériorité dignes d'admiration. Et si moi je ressentais de l'admiration, a fortiori les autres, qui n'avaient pas d'abord éprouvé des réticences. Je me demandai évidemment jusqu'à quel point la jeune fille ardente l'admirait aussi, mais je n'eus pas longtemps à attendre pour connaître la réponse.

Cela se passa un matin dans mon bureau. La jeune fille ardente et moi étions assis l'un en face de l'autre, en train de discuter un projet de pièce radiophonique dans laquelle elle allait jouer. Le jeune homme aux yeux de braise entra tout d'un coup. Pas pour me voir, mais pour s'adresser à elle :

" Je vais vous prédire l'avenir!", déclara-t-il en saisissant sa main et en examinant les lignes de la paume. Ce n'est pas seulement son insolence qui m'agaça : tout en détaillant la main de la jeune fille, son coude heurtait visiblement sa poitrine. Plus intrigant encore, la jeune fille soumise à ces assauts acceptait cela en riant de joie.

«Exactement ce primitif de Kawista!», pensai-je lorsqu'ils m'eurent quitté.

À partir de ce moment-là, je compris que ma relation avec la jeune fille ardente avait pris fin. Comment sa valeur en tant que jeune fille destinée à être mon épouse n'aurait-elle pas diminué à mes yeux, après un tel épisode ? Le chef de service fit bien un effort pour nous réconcilier, mais sans succès. Sa mère demandait souvent pour quelle raison je ne venais plus chez elle, mais je répondais évasivement. 
Plus tard, lorsque le bureau du Putera fut fermé et les Japonais chassés d'Indonésie, j'appris que la jeune fille ardente s'était mariée. Pas avec le jeune homme aux yeux de braise, ni avec l'un des employés du Putera qui s'intéressaient à elle. L'homme qu'elle épousa était un petit fonctionnaire, un ancien voisin qui lui était un jour venu en aide lorsque sa maison avait été inondée.

Plus tard encore, alors que j'avais une famille et habitais à Jakarta, j'eus des nouvelles par ma mère : « On la voit souvent vendre de l'or et des bijoux au mont de piété », dit-elle.

Ce que je ne pouvais pas prévoir, c'est le destin du jeune homme aux yeux de braise. Pendant la révolution d'août 1945, j'entendis dire qu'il avait encore fait la cour à la jeune fille, quand il habitait à Yogyakarta, mais lorsqu'il apprit qu'elle était mariée, il se sentit dépité et abandonna.

Par la suite, je l'ai fréquemment rencontré lors de réceptions dans des ambassades étrangères. Les invités dans ces lieux ne sont pas n'importe qui, vous vous en doutez. Il était alors devenu un personnage important, un politicien dirigeant un parti. Une chose que je ne peux pas oublier, c'est que, chaque fois qu'il me présentait à un étranger dans ces réceptions, il employait toujours la même phrase anglaise : "When I prepare the revolution, he prepares his Tambera », disait-il.

Il semblait avoir une telle admiration pour mon Tambera que je lui demandai un jour : "Vous aimez mon Tambera parce que vous aimez le personnage de Kawista, n'est-ce pas?»

«C'est vrai, dit-il. Kawista est un homme fort. »

Je ris en moi-même - de plaisir, en pensant qu'il ignorait que le personnage qu'il encensait était inspiré de sa propre personne.

J'avais en fait appris de divers amis qu'il désirait s'entretenir avec moi, afin d'établir une collaboration entre nous, lui le politicien et moi l'écrivain. Mais les faits avaient abondamment prouvé que les politiciens ne s'intéressaient généralement aux écrivains que dans le but de les dominer pour les utiliser. Et je n'étais certainement pas prêt à être dominé et utilisé par quelqu'un d'autre. C'est pourquoi, lorsque je le rencontrais dans ces réceptions, je ne lui parlais pas plus que nécessaire.

Mais l'histoire décida tout de même de nous rapprocher, lui et moi. Cela débuta le jour où $\mathrm{j}$ 'appris qu'il allait prononcer un discours dans un immense meeting, devant des milliers de personnes. Je désirais écouter son discours, mais c'est surtout parce qu'on était dimanche que j'allai y assister avec mon fils et m'assis au milieu de la foule en grignotant des cacahuètes. À la fin du meeting, les spectateurs, couverts de sueur, se bousculèrent pour sortir. Mon fils et moi aussi étions en nage. Tout à coup, j'entendis le klaxon d'une voiture, juste derrière moi. La foule se rangeait pour libérer le passage et nous fîmes de même. Mais à mon grand étonnement, une fois arrivé à ma hauteur, le véhicule s'arrêta. C'était une voiture bleue, dont le numéro indiquait qu'elle 
appartenait à un ministre. Et plus étonnant encore, la porte s'ouvrit, et le passager, le jeune homme aux yeux de braise qui venait de parler, sortit la tête et dit : « Tuy, entrez donc ! »

Sans réfléchir, je pénétrai donc dans la voiture avec mon fils et m'installai à côté de lui. C'est là qu' eut lieu, entre lui et moi, une conversation à cœur ouvert, principalement sur notre vie de famille, la santé de la mienne comme celle de la sienne. Il nous conduisit jusqu'à un endroit où je trouverais facilement un minibus pour le quartier où j'habitais.

Quelques mois plus tard, ma femme et moi avons reçu une invitation pour un congrès des arts et des lettres dont il était président. Sans trop réfléchir, nous avons décidé d'y aller, avec l'idée, évidemment, que nous allions écouter plus que participer. J'ai prêté attention à son discours mot par mot, phrase par phrase. Il y avait encore beaucoup d'agitation politique, mais aussi quelque chose qui me toucha - une formule originale ou une citation, je ne sais pas :

« Si la politique est le cerveau du parti, la littérature est le coeur du parti. »

À la fin de la séance, je suis allé vers lui et je lui ai demandé :

« Etes-vous certain que politiciens et écrivains puissent travailler ensemble?»

Il me tendit la main en questionnant à son tour :

«Et vous, croyez-vous ou non que nous pouvons collaborer?»

Je me bornai à rire en lui serrant la main.

Depuis ce moment-là, nous avons eu fréquemment des conversations de fond sur l'art et la littérature. Il me demanda un jour : « Pourquoi ne finissezvous pas votre Tambera?»

« Il est fini », répondis-je.

« Mais j'ai entendu dire que, dans la version soundanaise, l'histoire est très longue et se termine par la vengeance de Tambera sur les Hollandais, alors que dans la version indonésienne, il n'y a pas de vengeance. Cela ne veut-il pas dire que la version indonésienne n'est pas terminée ?»

« Le problème, c'est le lieu et le moment où j’écris », répondis-je. « Comme vous le savez, j'ai écrit Tambera en soundanais du temps des Indes néerlandaises, alors que j'avais les Hollandais sous les yeux, à une époque pleine de romantisme, tandis que j'ai écrit le Tambera indonésien pendant l'occupation japonaise, quand les Hollandais n'étaient plus là, quand le mode de vie 'romantique' commençait à disparaître et même que nous tous étions obligés d'afficher un 'visage ardent', au point que les jeunes filles que se disputaient les garçons ne pouvaient échapper au devoir du 'visage ardent'. Vrai ou non?»

Il a ri et même ri aux éclats, au souvenir sans doute de la jeune fille ardente du Putera, autrefois.

Notre dernière rencontre eut lieu, en août 1965, à l'hôtel Duta Indonesia, où je donnais une causerie. J'eus à peine terminé qu'il monta sur la scène et 
me donna l'accolade, puis il m'invita à m'asseoir à côté de lui. « J'entends dire que vous avez récemment souffert d'une maladie de foie », dit-il.

« C'est vrai », répondis-je.

«Vous savez que c'est une maladie sérieuse ? Que diriez-vous de vous faire soigner à l'étranger?»

Je n'avais pas encore répondu, je réfléchissais, qu'un de ses amis assis derrière nous est intervenu : « Votre femme vous accompagnerait à l'étranger. »

« Oui, bien sûr », confirma le jeune homme aux yeux de braise, « vous iriez vous soigner à l'étranger avec votre femme. Qu'en dites-vous ?»

« Très bien », dis-je.

Dès lors, ma femme et moi nous sommes préparés au départ, mais au dernier instant, on nous annonça que ma femme ne partait pas. Elle se mit en colère et moi-même, je faillis annuler mon départ, tant j'étais contrarié.

Deux jours après mon arrivée à l'étranger, je compris pourquoi ma femme devait rester sur place : elle devait s'occuper des enfants à la maison. Pour quelle raison ? Parce que ce jour-là, en Indonésie, se produisit l'événement sanglant que l'on a appelé « l'Affaire du 30 septembre », la catastrophe qui a fait des centaines de milliers de victimes, y compris des écrivains, des artistes et des politiciens, dont le jeune homme aux yeux de braise lui-même. Quand la nouvelle parvint à mes oreilles, j'étais dans un lit, à l'hôpital. Je ne pus retenir mes larmes, conscient que l'homme qui avait tout d'abord suscité mon antipathie avait, pour finir, fait son possible pour me sauver du désastre.

Bien sûr, vous vous demandez maintenant qui est donc le jeune homme aux yeux de braise? Son nom d'origine, celui que lui ont donné ses parents, franchement, je ne le connais pas. Mais celui qu'il avait créé, qu'il m'avait annoncé autrefois et qu'il avait porté jusqu'à son dernier jour est Dipa Nusantara Aidit. Pensez seulement à ce que vous inspire un nom dans lequel Dipa est emprunté à Dipanegara, le héros d'antan, et Nusantara renvoie à l'immensité du territoire de l'Indonésie actuelle !

"What's in a name? ", a dit Shakespeare.

L'homme qui crée son propre nom selon ses pulsions profondes, parce qu'il n'est pas satisfait du nom que lui ont donné ses parents, celui-là, pour sûr, possède pour le moins un talent de création poétique. Dipa Nusantara Aidit n'est certainement pas quelqu'un que l'on pourrait exclure de cette catégorie.

« Enfant d'une époque qui fera naître une époque nouvelle », ainsi dit un vers de sa composition.

Comme on le sait, « l'enfant d'une époque », dans ce poème, est le parti qu'il dirigeait, le Parti communiste d'Indonésie. Mais un poème, quoi qu'il en soit, reflète l'âme de son créateur, il est une image de sa personnalité. Pour moi, je peux affirmer qu'il était l'enfant de l'époque japonaise, un Indonésien dont la psychologie avait été forgée par l'occupation japonaise.

Que, dans son effort pour atteindre l'idéal exprimé dans les mots « qui fera naître une époque nouvelle », il ait rencontré la tragédie, pour moi, cela 
me rappelle involontairement la tragédie de Kawista dans mon propre roman.

Le personnage énigmatique n'est donc personne d'autre que D.N. Aidit. Le portrait initial qu'en fait Utuy dans cette nouvelle, alors qu'Aidit n'a que vingt ans (et Utuy 23), n'est guère flatteur : arrogant, impoli, impétueux, " primitif », bien qu'en même temps courageux et impressionnant. Leurs relations s'améliorent par la suite, cependant, et Utuy exprime, pour une période située environ vingt-deux ans plus tard, du respect, des intérêts communs et même une certaine sympathie. Le trait le plus frappant de ce portrait est l'expression " jeune homme aux yeux de braise » (si pemuda belotot), car ce sont en effet les yeux d'Aidit qui frappent en premier lieu dans chacune de ses photographies (voir fig. 2).

Utuy mentionne qu'Aidit était un favori de Bung Hatta (anak mas Bung Hatta). Il semble qu'il y ait là une exagération; peut-être une aura de légende entourait-elle déjà la personne d'Aidit à l'âge de 20 ans. Hatta et Aidit ont probablement fait connaissance au Gedung Menteng 31, où le premier faisait partie des instructeurs et le second des élèves, mais ils n'ont jamais été intimes ${ }^{11}$. Le dirigeant du parti communiste qui, semble-t-il, pouvait s'enorgueillir d'avoir été le " chouchou » de Hatta était M.H. Lukman, qui l'avait connu alors qu'il était encore enfant, à Boven Digoel, où le père de Lukman était déporté et où Hatta passa l'année 1935. C'est alors, selon la fille de Lukman, que celui-ci fut traité par Hatta comme son propre fils (voir Tatiana Lukman, 2014 : 172).

Il est nécessaire de noter que le texte ci-dessus est un récit inspiré de faits réels, mais qu'il ne vise pas pour autant à l'authenticité ${ }^{12}$ : il s'agit d'un texte de nature littéraire, composé de façon méticuleuse et qui manipule la réalité, pour la raison sans doute qu'à l'époque où il fut écrit, Utuy désirait renier son alliance passée avec le parti communiste. Le récit, en effet, donne l'impression que, si «l'homme aux yeux de braise » est ministre et chef d'un parti politique, Utuy, lui, est un simple écrivain qui ne désire adhérer à aucun parti ; et c'est donc par estime et par sympathie qu'Aidit lui offre de se soigner en Chine aux frais du parti communiste. La réalité est tout autre. En 1965, lorsque Aidit lui proposa d'aller en Chine, Utuy faisait partie depuis six ans du comité directeur du Lekra ${ }^{13}$.

11. Sobron Aidit, cependant, note (2003: 21) qu'Aidit était un « élève apprécié » de Hatta. Watson (2006: 94) apporte un complément d'information à ce sujet.

12. Le faux-titre du recueil Di Bawah Langit Tak Berbintang (2001) porte en sous-titre « Mémoires, autobiographie, roman ? Ce qui importe, c'est de le lancer, afin qu'il scintille dans l'obscurité de la nuit ». Ce sous-titre ne s'appliquait à l'origine qu'au quatrième récit (éponyme), mais il qualifie parfaitement la nature de tout le volume.

13. Créé en 1950 par des dirigeants du Parti communiste, le Lekra (Lembaga Kebudayaan 


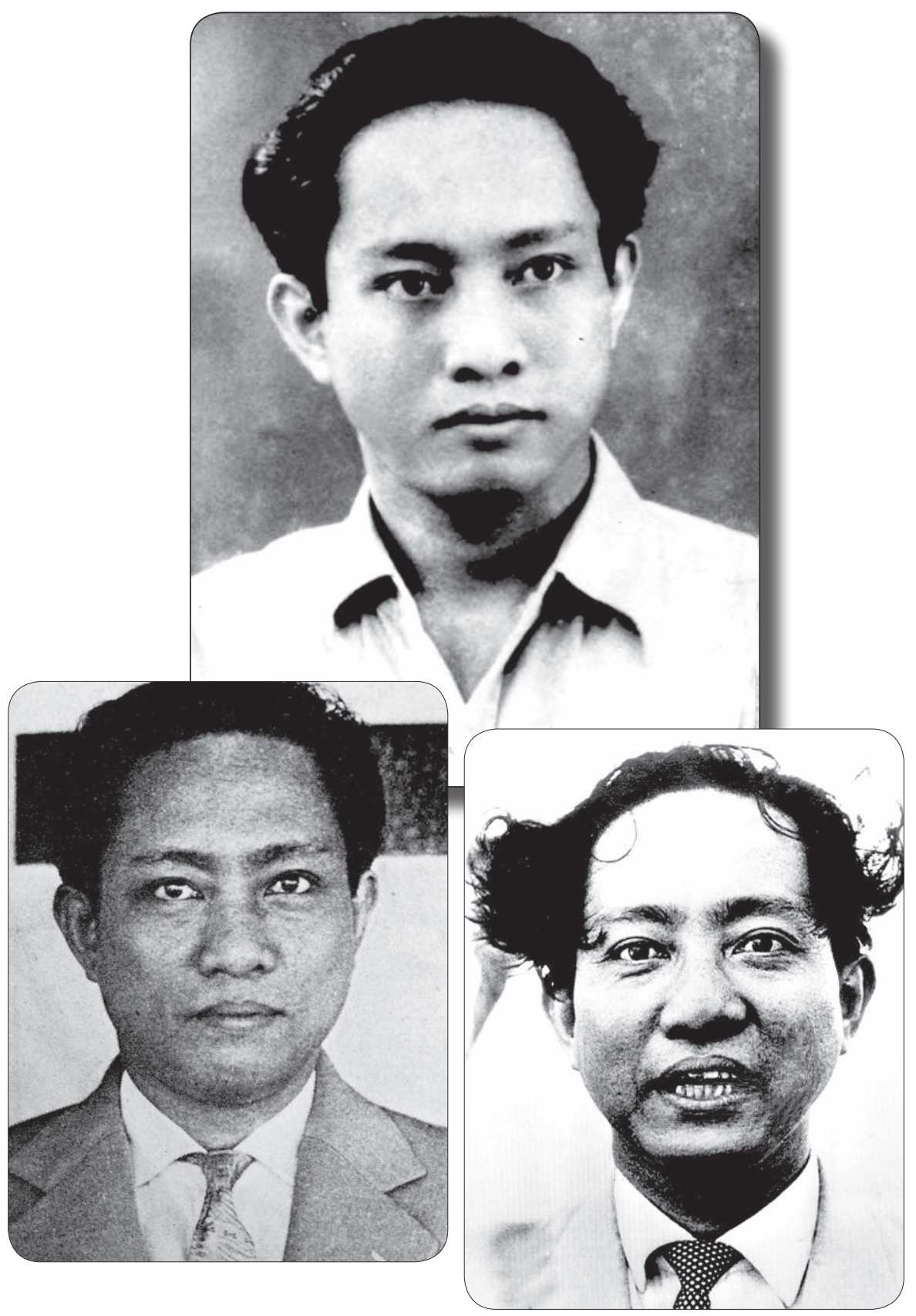

Fig. 2 - Trois photos de « l'homme aux yeux de braise». 
Il est par ailleurs improbable que son envoi à Pékin se soit décidé en quelques minutes, dans un hôtel, au mois d'août 1965. Une telle décision a sans doute donné lieu à des discussions dans un bureau du Parti ou du Lekra ${ }^{14}$. La manipulation est-elle littéraire ou politique? Quoi qu'il en soit, les histoires du recueil ne sont pas des souvenirs candides. La dimension littéraire des textes autobiographiques - la sélection des faits, leur présentation et leur composition d'ensemble - a été ignorée jusqu'à présent, notamment dans l'analyse de Watson (2006), par ailleurs extrêmement lucide et érudite, si bien que les souvenirs d'Utuy sont généralement tenus pour authentiques. De nombreux indices contredisent ce point de vue.

Selon Ajip Rosidi (2001: 8-9), Utuy fut approché par le PKI dès 1957, et il fit partie, cette année-là, de la délégation de l'Union des Écrivains d'Asie et d'Afrique au congrès de Tachkent (alors capitale de la République soviétique d'Ouzbékistan), et l'année suivante au congrès de Moscou. Il refusa tout d'abord l'offre d'entrer à la direction du Lekra, mais dès janvier 1959, il faisait partie de la Direction centrale élue lors du premier congrès de l'association ${ }^{15}$. On ne sait pas avec certitude, par contre, s'il était membre du Parti. Au début du récit Di Bawah Langit, le narrateur raconte que, lorsqu'il partit pour Pékin, il était membre du Parti depuis six mois (2001 : 80), mais plus loin il précise qu'il n'était en réalité que candidat.

Pour Ajip Rosidi (2001 : 8-9), Utuy faisait partie de ces artistes qui furent happés par le PKI ou le Lekra du fait d'amitiés ou de compromissions, mais qui avaient une connaissance superficielle et erronée des thèses communistes. Ajip Rosidi est revenu sur ce thème à plusieurs reprises, à propos d'Utuy et d'autres écrivains et artistes qui ont rejoint le Lekra ou le PKI, sans comprendre, affirme-t-il, la nature et la portée de leur geste sur le plan politique. Ceci est même l'un des thèmes majeurs de son roman Anak Tanah Air paru en 1985. Dans un article de 1964, Ajip a relaté qu'Utuy, de son propre aveu, n'aimait pas lire et ne finissait jamais un livre parce que son imagination l'emportait toujours avant la fin. Par ailleurs, il a toujours manifesté, jusqu'à la fin des années 50, du dégoût pour la politique et pour les politiciens, qu'il traitait de « bouffons ». À partir de son adhésion au Lekra, en 1959, il s'est mis à donner des conférences, à participer à des réunions et à écrire des essais. Il fit preuve soudain d'un grand intérêt pour la politique et pour les grandes théories, et il se mit à utiliser des slogans et un jargon politique. Il affirma désormais que la politique devait commander (harus menjadi panglima) les arts et les lettres.

Rakyat, Institut de la culture populaire) rassembla les écrivains de gauche indonésiens sous la bannière du réalisme socialiste et précipita la confrontation avec les écrivains libéraux ou apolitiques jusqu'en 1965, date à laquelle il fut dissous, en même temps que le PKI.

14. Alex Supartono (2001: 133) donne des détails sur le processus bureaucratique du départ d'Utuy. 15. Le Laporan Kebudajaan Rakjat (Rapport sur la culture populaire) de 1959, p. 85, donne la liste du Pimpinan Pusat Lekra élu le 28 janvier 1959, au cours du Kongres Nasional Ke-I Lekra : Utuy est le quarantième des 41 membres. 
Dans les conversations privées, il aimait se qualifier de "ur-communiste » (uhr-komunis atau komunis-purba). Ajip note encore qu'Utuy appliquait de façon erratique à la littérature soundanaise des théories fantaisistes sur les dimensions sociale et politique de la littérature (Rosidi, 1964/2015 : 20-23). Il conclut malheureusement : « Ce qui est triste, c'est qu'Utuy, clairement, est devenu membre du bureau du Lekra et a adopté une conception communiste, non pas à la suite d'une évolution de sa conscience spirituelle, politique et sociale arrivée à son terme, mais parce qu'il s'est prostitué, ni plus ni moins. » (Idem, p. 29) ${ }^{16}$.

Le monde des lettres, dans les années 1950, était soumis à une forte tension, du fait de la polarisation provoquée par l'essor des idées socialistes et communistes dans le monde politique. Certains écrivains et artistes optèrent pour le camp communiste par faiblesse ou par opportunisme, comme le décrit Ajip Rosidi, mais d'autres, la majorité certainement, cédèrent à la passion politique qui envahissait l'espace social et à l'enthousiasme suscité par les idéaux populistes largement diffusés par le parti communiste et ses multiples antennes. Utuy a exprimé, dans ses oeuvres des années 50, un désenchantement vis-à-vis de l'ordre social. L. Santa Maria, au terme d'une longue analyse, concluait : « nous voudrions noter que l'œuvre de Sontani ne peut être que le produit d'un écrivain insatisfait de la société dans laquelle il vit effectivement » $(1965: 257)$.

H. Aveling, de la même manière, note qu' ' il y a, dans les écrits de Sontani, une très nette suggestion que tout pouvoir politique est cruel et arbitraire régime néerlandais, Révolution et forces capitalistes bureaucrates tout aussi bien » (1979 : 13). Utuy n'avait peut-être pas la fibre politique, mais il avait une opinion sur la société dans laquelle il vivait. Dès 1952, interviewé par Pramoedya Ananta Toer, il déclarait : « Je voudrais que l'on puisse croire à nouveau en l'homme. Mais cette foi, aujourd'hui, a disparu. Il ne reste qu'un désir, une curiosité. Comment voulez-vous croire en l'homme, quand l'homme que vous cherchez n'existe pas ? Et quand vous vous trouvez au milieu d'animaux, comment pouvez-vous espérer rencontrer des hommes ? $\gg{ }^{17}$. Pram conclut de cet entretien que, dans le milieu des écrivains indonésiens, Utuy avait atteint le faîte du pessimisme.

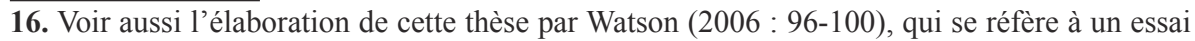
d'Ajip paru en soundanais.

17. " Aku ingin orang bisa mempercayai manusia lagi. Tetapi kepercayaan pada manusia sekarang ini sudah pudar. Yang ada hanya keinginan-tahu, nieuwsgierheid. Bagaimana engkau bisa mempercayai manusia, kalau manusia yang kauhendaki itu tidak ada. Dan kalau engkau berada di lingkungan binatang, bagaimana bisa engkau mengharapkan dapat bersinggungan dengan manusia? », dans Pramoedya Ananta Toer, «Berkenalan dengan Utuy Tatang Sontani », Siasat, 7 Sept. 1952, cité par Aveling, 1979 : 25. Cette citation se trouve dans Supartono (2001: 133. 144), avec d'étranges variantes. 
Il est probable, comme l'affirme Ajip Rosidi, qu'Utuy n'était pas familier des thèses politiques, économiques et sociales du communisme, mais son emploi du terme «ur-communiste » laisse penser qu'il concevait son entrée au Lekra et son adhésion à certaines thèses communistes relatives à la littérature, non comme le résultat de lectures ardues et de connaissances nouvelles, mais $\mathrm{du}$ fait de l'adéquation entre une vague conception du communisme comme l'outil d'une plus grande justice sociale et d'une reconnaissance de la culture du peuple, et son aspiration personnelle à une société plus égalitaire et plus harmonieuse. Utuy a publié dans Harian Rakyat et Bintang Timur, deux organes du PKI, plusieurs articles inspirés par les thèses du réalisme socialiste (v. Aveling, 1979 : 55), ainsi que deux pièces résolument socialistes, Si Sapar et Si Kampeng. Il est clair qu'il a adhéré aux thèses et aux mots d'ordre du Lekra et a tenté de les mettre en oeuvre. Il se trouve par exemple, dans le récit « Haru yang tak kunjung kering » (L'inaltérable émotion, le deuxième texte de Di Bawah Langit) un embryon d'analyse sociale intéressant à cet égard. Dans des conférences données en 1963 et 1964, il se décrivit comme un écrivain venu de la classe petit-bourgeoise qui avait choisi de s'identifier aux travailleurs, aux ouvriers et aux paysans, et il se déclara convaincu que son oeuvre devait servir la classe des travailleurs (Aveling, 1979 : 45-46). Il semble, par contre, qu'il ne fut jamais reconnu comme un écrivain communiste par ses pairs du parti. À la fin de 1965, alors précisément qu'il était en passe de quitter la scène, il était qualifié, dans Bintang Timur, " d'écrivain noncommuniste » (Aveling, 1979:46).

Beaucoup plus tard, vers 1976, Utuy renia catégoriquement son allégeance communiste. A Waloejo Sedjati, il confia : «En réalité, je ne suis pas communiste. Je ne connais pas, je ne comprends pas ce qu'est le communisme. Je suis entré au Lekra parce que j'étais proche de Nyoto. Pour moi, Nyoto était un être beau (orangnya indah), un politicien en même temps qu'un artiste. De là, je considérai qu'il y avait de la beauté dans le communisme. Mais maintenant, je n'aime pas le Parti et pas plus le communisme. (...) Aussi, quand j'ai organisé mon installation ici, j'ai demandé à ne pas être aux mains du Parti. C'est l'Union des écrivains soviétiques qui s'est occupée de moi, en la personne de Sikorsky. » (2013 : 258). Et encore : « Le Parti, ah! Une organisation qui enchaîne, une tyrannie qui écrase la liberté de créer. » (p. 260).

Ces propos sont pratiquement identiques à ceux que tient le narrateur du récit Di Bawah Langit s'adressant à une femme qui est sa confidente (2001: 81). Il est clair cependant qu'il s'agit là de déclarations formulées après dix ans d'exil et de souffrances, dix ans d'un sort haï causé par son appartenance au Lekra, des déclarations qui ne disent donc rien de ses sentiments et ses opinions entre 1959 et 1965.

Outre un portrait d'Aidit, le récit What's in a name ci-dessus contient une interprétation implicite du « coup » de 65 pour le moins surprenante. 
Le narrateur, en effet, conclut qu'Aidit, en l'envoyant en Chine, a voulu lui sauver la vie, tandis qu'il maintenait sa femme à Jakarta, afin qu'elle s'occupe de leurs enfants. Ceci implique qu'Aidit savait que le coup était imminent (ceci est aujourd'hui à peu près établi), mais qu'il savait aussi qu'Utuy risquait sa vie s'il restait à Jakarta et qu'il ne pourrait pas rentrer en Indonésie s'il était à l'étranger. Autrement dit, Aidit savait que le putsch allait entraîner une purge sanglante du PKI.

Cette idée qu'Aidit avait prévu le cataclysme semble tout simplement invraisemblable : Aidit, certainement, aurait fait son possible pour prévenir le putsch s'il avait pu penser que le PKI en ressortirait anéanti. Et pourtant, Utuy n'est pas le seul à avoir exprimé cette hypothèse. Dans sa communication à un colloque qui s'est tenu à Paris en 2016, Ibarruri Sudharsono, la propre fille de D.N. Aidit, a raconté :

« À ce moment de l'histoire de l'Indonésie, le Parti communiste d'Indonésie représentait, par son importance, le troisième parti communiste au sein du mouvement communiste international et le plus important hors du camp socialiste. Après les déjà terribles répressions de Madiun en 1948, dès les années suivantes, le PKI s'est reconstitué pour devenir aux élections de 1955 la quatrième organisation politique du pays. En 1965, les Indonésiens avaient l'impression que bientôt les communistes allaient assumer la direction du pays. Le 17 août 1965, j’ai écouté le discours du Président Sukarno avec des milliers de jeunes réunis devant le palais présidentiel à Jakarta, puis j'ai participé au défilé, dans les rangs du mouvement de la jeunesse communiste, jusqu'au siège du Parti. Ce soir-là mon père, DN Aidit, qui était le président du PKI, y a prononcé un discours dont je n'oublierai jamais les termes, tant je ressens aujourd'hui encore l'impression étrange avec laquelle je l'ai perçu ce jour-là. S'il a rappelé que le Parti avait survécu deux fois à la terreur des forces réactionnaires et de leurs exécutants militaires ou miliciens, en 1926, puis en 1948, il a annoncé, ce qui m'a sidérée à cet instant, qu'une troisième terreur allait nous frapper, pire que les précédentes dans la cruauté. Et son discours ne s'est pas terminé sans l'expression néanmoins de sa confiance dans la capacité de la génération future à en tirer toutes les leçons, pour relever encore le Parti, le rendre plus fort et en mesure de diriger la révolution nécessaire à l'émancipation et au développement de notre peuple. Tous ses mots ont sonné de manière si étrange dans l'ambiance euphorique de ce moment, sans que nous en percevions la portée prémonitoire. Et quelques mois plus tard l'atroce réalité annoncée donnait chair à son discours. »

Ibarruri Sudharsono avait déjà relaté ce souvenir dans ses mémoires (Alam, 2006 : 93-94). Un troisième témoignage, plus ambigu cependant, vient de Waloejo Sedjati, qui se trouvait à Pyongyang à la fin septembre 1965. Lors d'une cérémonie organisée à l'occasion de la visite d'une délégation de quarante membres du parlement indonésien présidée par le ministre Chaerul Saleh (et qui repartit pour Jakarta précisément le 29 septembre), un membre du comité central du PKI faisant partie de la fraction communiste de la délégation exposa la situation en Indonésie en ces termes : Bung Karno était gravement malade, et un Dewan Jendral («Conseil des Généraux ») composé de généraux de droite de l'Armée de Terre préparait un coup d'Etat pour le 5 octobre ; ils élimineraient (menghancurkan) le PKI avant de renverser Soekarno (2013 : 169). 
La thèse d'un anéantissement du Parti prévu et assumé par les dirigeants du PKI est sans doute un fantasme, mais qu'il convient d'inscrire dans la perception des événements par certaines des personnes qui en ont subi les conséquences. D.N. Aidit a ainsi le rôle d'un prophète qui sacrifie son peuple dans la promesse d'un avenir meilleur. Cette idée rejoint une croyance nousantarienne, très ancienne et très répandue, qu'un cataclysme destructeur est parfois nécessaire à la rédemption d'une communauté ${ }^{18}$. Rédemption, en l'occurrence, comme cela est illustré par le cas d'Aceh, n'implique pas une faute qu'il faudrait expier; il s'agit de permettre un renouvellement radical, une mutation à un stade supérieur. Une telle interprétation de la décision d'Aidit est donc en quelque sorte optimiste : elle garantit un futur glorieux ; elle présente les événements comme annoncés et délibérément acceptés, et non subis passivement ; elle fait en outre d'Aidit un martyr.

Le portrait de «l'homme aux yeux de braise » donne lieu à une comparaison répétitive avec le roman Tambera, qui a une signification allégorique relative à l'histoire nationale, et qui acquiert ici une signification symbolique nouvelle : l'opposition entre deux tempéraments et deux destins. Du jour où ils font connaissance, le narrateur, c'est-à-dire Utuy tel qu'il se met lui-même en scène, qui s'identifie à son héros Tambera, s'intéresse au jeune homme qui, parce qu'il ressemble à Kawista, en devient le modèle dans la version indonésienne qu'il est en train d'écrire. L'action du roman se situe dans l'île de Banda autour de 1600 et brode de façon fantaisiste sur la domination que les Hollandais imposent par les armes aux habitants de l'île. Hormis ce contexte général, le roman n'a rien d'historique ${ }^{19}$. Il est surprenant qu'Utuy s'identifie au personnage de Tambera parce que celui-ci, dans la version indonésienne, est un jeune homme rêveur, sentimental et immature qui n'agit que par égoïsme et qui méprise sa propre nation, au point de la trahir. Mais en réalité, on ne peut en juger sur la version indonésienne, car celle-ci, bien que nettement plus longue que la version originale soundanaise, est inachevée : Utuy prévoyait de publier un deuxième volume (cf. Ajip, 1964/2015 : 18 ; Jassin $1967: 175$; Santa Maria, 1965 : 247), dans lequel le personnage allait peut-être évoluer de façon radicale. Notons au passage que la comparaison n'a jamais été faite entre les deux versions, soundanaise et indonésienne, du roman.

Une autre opposition entre le narrateur et Aidit se trouve dans le nom qu'ils ont choisi l'un et l'autre : Utuy révèle ici que le nom sous lequel il est connu (Utuy Tatang Sontani) est un nom qu'il s'est choisi lui-même. Ceci était totalement ignoré jusqu'à présent, même par exemple d'Ajip Rosidi, qui a été un ami proche d'Utuy dans les années 50 et 60 , et qui a une connaissance

18. Cette notion a été invoquée lors du tsunami d'Aceh, en 2004, et de plusieurs catastrophes naturelles, notamment des éruptions volcaniques. Voir par exemple Lapian, 1987 ; Wieringa, 2010.

19. Voir Santa Maria (1965 : 247) et Aveling (1967). 
encyclopédique de la littérature de l'époque. Personne, donc, ne connaît le nom d'origine d'Utuy. Sa voisine, dans la première nouvelle de Di Bawah Langit, l'appelle « Jun »; sa logeuse à Bandung, dans la deuxième nouvelle, l'appelle « Dadang ${ }^{20}$. Tatang pourrait être une déformation de Dadang. Sontani est le nom du héros d'un roman inspiré par le bagne de Boven Digoel intitulé Minggat dari Digoel (Les Évadés de Digoel, cf. Supartono, 2001 : 135). Le nom Utuy Tatang Sontani, dit le narrateur dans la nouvelle ci-dessus, est « mélodieux »; c'est un nom de poète. Celui que s'est choisi Aidit, au contraire, Dipa Nusantara, est ambitieux et chargé d'idéologie ; c'est un nom de guerrier. Le nom Dipa n'est pas emprunté à celui de Dipanagara, le héros de la Guerre de Java (1830-35), comme l'avance Utuy, mais au mot javanais dipa, «lampe ». Il est vrai que le nouveau nom d'Aidit, signifiant « Lumière de l'Archipel », formait un calque de celui de Dipanagara, « Lumière du royaume ». Trois des frères cadets d'Aidit, respectivement Murad, Sobron et Asahan, ont apporté un témoignage sur cette question de nom. Par ordre chronologique de publication, Sobron, dans un livre de souvenirs sur son frère $(2003: 11,20)$, relate que celui-ci s'appelait à l'origine Achmad Aidit et était familièrement appelé Amat. À l'âge de 17 ans, en 1940 donc, il décida de changer de nom et choisit celui de Dipa Nusantara. Il reçut l'autorisation de leur père, et la modification fut enregistrée chez un notaire de Batavia. Murad, dans un autre ouvrage consacré à Aidit (2005: 67-68), rapporte les mêmes faits, mais d'une part précise que Aidit voulut changer de nom afin de brouiller les pistes sur son origine, car il risquait d'ètre recherché par la police, et d'autre part avance que leur père, tout d'abord opposé à cette modification, ne céda qu'après de longues négociations. Asahan, quant à lui, a affirmé dans le roman Alhamdulillah $(2006$ : 286) que le nom Dipa Nusantara a été donné à son frère à sa naissance par leur père, mais Asahan est connu pour avoir introduit des éléments de fiction dans ses écrits autobiographiques, y compris à propos de sa propre famille.

Utuy signale, dans le récit, qu'un troisième personnage portait un nom inventé : Kelana Asmara, son assistant à la Putera, jeune poète et son "plus proche compagnon de lutte ", dont le nom revient quatre fois dans le texte. Kelana Asmara ("le vagabond de l'amour ») est en effet l'un des nombreux pseudonymes de A.S. Dharta (1924-2007), qui sera, après l'époque du récit, l'un des fondateurs du Lekra et passera treize ans en prison après 1965.

Le roman Tambera oppose deux attitudes face à l'occupation étrangère : le jeune Tambera, faible et égoïste, s'engage par amour dans l'armée des envahisseurs, tandis que Kawista, brutal et arrogant, se dévoue corps et âme à la cause de sa nation. Dans ce contexte, comment ne pas voir une métaphore dans

20. Selon Alex Supartono (2001 : 136), sa femme l'appelait Jun, et ses amis, au début des années 40, Dadang. 
le fait que Kawista conduit son peuple à la destruction et lui-même à l'exil ? Aidit également, comme le suggère la dernière phrase du récit, s'est montré impétueux, présomptueux et déraisonnable, et a conduit le PKI au désastre ${ }^{21}$.

\section{BIBLIOGRAPHIE}

Aidit, Asahan. Alhamdulillah: Roman Memoar. Jakarta: Lembaga Sastra Pembebasan, 2006.

Aidit, Murad. Aidit Sang Legenda. Jakarta : Panta Rei, 2005.

Aidit, Sobron. Aidit : Abang, Sahabat dan Guru di Masa Pergolakan. Bandung : Nuansa, 2003.

Alam, Ibarruri Putri. Roman Biografis Ibarruri Putri Alam, Anak Sulung D.N. Aidit. Jakarta: Hasta Mitra, 2006. (Deuxième édition, Maumere, 2015.)

Aveling, Hary. Man and Society in the Works of the Indonesian Playwright Utuy Tatang Sontani. University of Hawaii, Southeast Studies Program, Southeast Asia Paper no. 13, 1979.

Aveling, H. « Seventeenth century Bandanese society in fact and fiction: Tambera assessed », Bijdragen tot de taal-, land-en volkenkunde, 123-3, 1967, p. 347-365.

Hill, David T. "Indonesian political exiles in the USSR », Critical Asian Studies, 46-4, Dec. 2014, p. 621-48.

Jassin, H.B. Kesusastraan Indonesia Modern dalam Kritik dan Esei. Djakarta: Gunung Agung, 1967, vol. I.

Lapian, A.B. "Bencana alam dan penulisan sejarah (Krakatau 1883 dan Cilegon 1888)", in T. Ibrahim Alfian dkk. (eds.), Dari Babad dan Hikayat sampai Sejarah Kritis: Kumpulan Karangan Dipersembahkan kepada Prof. Dr. Sartono Kartodirdjo, Yogyakarta: Gadjah Mada University Press, 1987, p. 211-231.

Laporan Kebudajaan Rakjat. Dokumen (I) Kongres Nasional Pertama Lembaga Kebudajaan Rakjat, Solo, 22-28 Djanuari 1959. Djakarta: Lembaga Kebudajaan Rakjat, 1959.

Lombard, Denys. Histoires courtes d'Indonésie, soixante huit «tjerpén »(1933-1965). Paris : EFEO, 1968.

Lukman, Tatiana. Panta Rhei: Tidak Ada Pengorbanan yang Sia-Sia, Air Sungai Digul Mengalir Terus. Yogyakarta: Pustaka Sempu, 2014.

Rosidi, Ajip. Membitjarakan Tjeritapendek Indonesia. Djakarta: Djambatan, 1959.

Rosidi, Ajip. «Utuy menindjau perkembangan sastra Sunda », Duta Masjarakat, 31 Mei, 7 \& 14 Juni 1964. Republié sous le titre «Utuy T. Sontani memandang sastera Sunda » dans Ajip Rosidi, Lékra bagian dari PKI, Jakarta: Pustaka Jaya, 2015, p. 17-50.

Rosidi, Ajip. « Pengantar », in Sontani, 2001, p. 7-20.

Rosidi, Ajip. Hidup Tanpa Ijazah: Yang Terekam dalam Kenangan. Jakarta: Pustaka Jaya, 2008.

Santa Maria, Luigi. « Significato e problematico dell-opera di Utuy Tatang Sontani », Annali (Napoli), 15, 1965, p. 237-278.

Sedjati, Waloejo. Bumi Tuhan: Orang Buangan di Pyongyang, Moskwa, dan Paris (19602013). Jakarta: Kompas, 2013.

Sontani, Utuy Tatang. Di Bawah Langit Tak Berbintang. Jakarta: Pustaka Jaya, 2001.

Sudharsono, Ibarruri. " La Stratégie révolutionnaire de D.N. Aidit », Communication à la Semaine anti-coloniale, Paris, mars 2016, inédit.

Supartono, Alex. " Rajawali Berlumur Darah: Karya-Karya Eksil Utuy Tatang Sontani », Kalam Jurnal Kebudayaan, 18, 2001, p. 133-159.

21. Je désire exprimer mes plus vifs remerciements à Ibarruri Sudharsono et Ajip Rosidi pour avoir partagé avec moi leurs souvenirs et leurs opinions sur divers sujets traités dans cet article. Je remercie également Ade Pristie Wisandhani, Antonia Soriente et Pierre Chambert-Loir pour diverses ressources documentaires. 
Teeuw, A. Modern Indonesian Literature. The Hague: M. Nijhoff, vol. I, 1979.

Vigile (nouvelles, trad. Denys Lombard et al.). Jakarta: Forum Jakarta-Paris, 2009.

Watson, C.W. Of Self and Injustice: Autobiography and repression in modern Indonesia. Leiden: KITLV Press, 2006.

Wieringa, Edwin. "God speaks through natural disasters, but what does he say? Islamic interpretations in Indonesian tsunami poetry », in Arndt Graf et al. (eds.), Aceh: History, politics and culture, Singapore: ISEAS, 2010, p. 316-333. 